\title{
Local, atomic-level elastic strain measurements of metallic glass thin films by electron diffraction
}

C. Ebner ${ }^{1}$, R. Sarkar², J. Rajagopalan ${ }^{2,3}$, C. Rentenberger ${ }^{1}$.

1. Physics of Nanostructured Materials, Faculty of Physics, University of Vienna, Boltzmanngasse 5, 1090 Vienna, Austria.

2. Department of Materials Science and Engineering, School for Engineering of Matter Transport and Energy, Arizona State University, Tempe 85287, USA.

3. Department of Mechanical and Aerospace Engineering, School for Engineering of Matter Transport and Energy, Arizona State University, Tempe 85287, USA.

\section{Cite as:}

Ultramicroscopy 165 (2016) 51-58.

DOI: $10.1016 /$ j.ultramic.2016.04.004

\section{Abstract}

A novel technique is used to measure the atomic-level elastic strain tensor of amorphous materials by tracking geometric changes of the first diffuse ring of selected area electron diffraction patterns (SAD). An automatic procedure, which includes locating the centre and fitting an ellipse to the diffuse ring with sub-pixel precision is developed for extracting the 2-dimensional strain tensor from the SAD patterns. Using this technique, atomic-level principal strains from micrometre-sized regions of freestanding amorphous $\mathrm{Ti}_{0.45} \mathrm{Al}_{0.55}$ thin films were measured during in-situ TEM tensile deformation. The thin films were deformed using MEMS based testing stages that allow simultaneous measurement of the macroscopic stress and strain. The calculated atomic-level principal strains show a linear dependence on the applied stress, and good correspondence with the measured macroscopic strains. The calculated Poisson's ratio of 0.23 is reasonable for brittle metallic glasses. The technique yields a strain accuracy of about $1 \times 10^{-4}$ and shows the potential to obtain localized strain profiles/maps of amorphous thin film samples.

\section{Keywords}

Thin Films, Metallic Glass, Electron Diffraction Pattern, Amorphous Alloy, In-situ TEM.

(C) 2016. This manuscript version is made available under the CC-BY-NC-ND 4.0 license http://creativecommons.org/licenses/by-nc-nd/4.0/ 


\section{Introduction}

The measurement of elastic strain is important for the analyses of physical properties of materials, e.g. the electronic properties of semiconductors. The miniaturization of electronic components and the interest in nanostructures necessitates strain characterization on a local scale. Therefore, in recent years several new techniques have been developed to measure lattice strains of crystalline materials at nanometer scale resolution using transmission electron microscopy (TEM) methods [1,2]. The techniques used to obtain quantitative information are based on either (i) TEM imaging like high resolution TEM [3] and dark-field electron holography [4] or (ii) TEM diffraction like nano-beam electron diffraction [5] and convergent beam electron diffraction [6].

In the case of amorphous materials, elastic strain and large scale strain distribution have been measured only by the use of X-ray diffraction [7-10] even though metallic glasses have also been studied intensively by TEM methods due to their unique mechanical properties [11-13]. The strain determination using X-ray diffraction is based on measuring the deviation of the broad diffraction rings from circular symmetry. This diffraction-based method enables the determination of the atomic-level elastic strain of amorphous materials whereas the macroscopic strain contains anelastic contributions as well [14].

In this paper we show that TEM selected area electron diffraction (SAD) can also be applied to analyze atomic-level elastic strains of metallic glass thin films. Using this method, we obtain the elastic strain tensor from micrometer-sized regions of freestanding, amorphous TiAl film samples during in-situ TEM tensile deformation. In addition, the elastic properties of the sample are also calculated by taking the applied stress into account. The determination of elastic strain requires an accurate analysis of the SAD patterns, which is implemented as a plug-in (SAD-strain [15]) written for the GATAN ${ }^{\mathrm{TM}}$ Digital-Micrograph platform. By acquiring diffraction patterns at different sample locations, we show that strain profiles or strain maps can be obtained on a micrometer scale using this method.

\section{Atomic-level strain analysis of amorphous structure by electron diffraction}

The diffraction pattern of alloys with an amorphous structure typically consists of a few diffuse rings (cf. Fig. 1a). If the amorphous material is isotropic the elastic scattering intensity $I(\boldsymbol{q})$ is only a function of the magnitude of the scattering vector $\boldsymbol{q}$ and not of the direction. This rotational symmetry of the intensity distribution allows the integration along rings (over the azimuthal angle $\chi$ ) to obtain $I(q)$ (cf. Fig. 1b) as well as the structure function $S(q)$. The latter is calculated 
from $I(q)$ by taking into account the atomic scattering factors based on the composition of the material [9]. The circular symmetry of the SAD ring pattern (in the unstrained condition) is illustrated in Fig. 1c.

If the amorphous structure becomes anisotropic due to internal or external stresses, the diffraction pattern deviates from circular symmetry. This deviation from symmetry forms the basis for the atomic-level strain analysis of X-ray data, as suggested by Poulsen et al. [7]. During uniaxial tensile loading, the atoms will tend to move apart in the loading direction while in the transverse direction the atoms will move closer due to the Poisson's effect. These changes in real space distances lead to a shift of the intensity maxima in the diffraction pattern. The position $q_{1}$ of the first maximum is shifted to lower q-values in the tensile direction and to higher ones in the transverse direction. Hence, the overlay of a distorted SAD ring pattern with its rotated and inverted counterpart yields an image with a two-fold rotation axis only (cf. Fig. 1d). The relative change of the position of $q_{1}(\sigma, \chi)$ at a given stress $\sigma$ with respect to the unloaded position $q_{1}(0, \chi)$ can be used to calculate the atomic-level strain $\epsilon(\sigma, \chi)$ from reciprocal space analysis as a function of the azimuthal angle $\chi$ as reported in $[7,8,16]$ :

$\epsilon(\sigma, \chi)=\frac{q_{1}(0, \chi)-q_{1}(\sigma, \chi)}{q_{1}(\sigma, \chi)}$

In order to calculate small deviations from circular symmetry that represent strain, the positions of intensity maxima and the corresponding $q_{1}$-values have to be measured very accurately. Therefore, the centre of the diffraction pattern as well as the peak positions were measured with sub-pixel accuracy by data fitting procedures as explained below. These procedures are implemented as GATAN ${ }^{\mathrm{TM}}$ Digital Micrograph plug-ins, using the Armadillo library to easily handle matrices [17]. Additional features are implemented by calling script functions provided by the PASAD tools [18]. The algorithm performs the following steps of data analysis:

First, the algorithm tries to find the approximate centre of the SAD pattern. This is done by mirroring the image along its $\mathrm{x}$-axis and cross-correlating the so obtained image with the original one. The same process is then repeated after mirroring along the y-axis. The shift vectors between mirrored and un-mirrored images are then obtained from the maxima positions of the crosscorrelations, since the best correlation should be obtained when the amorphous rings overlap. In theory, one could simply flip the original image along the $\mathrm{x}$ - and $\mathrm{y}$-axis and obtain the centre from a single cross-correlation. But due to the beamstop needed to block the high intensity forward scattered beam this method becomes inaccurate. Therefore, by taking the mean of the two shift vectors obtained from the individual mirrored images, the influence of the beamstop is minimized and a first approximation for the centre $\left(\mathrm{x}_{0}, \mathrm{y}_{0}\right)$ is found. 
In the next step, the SAD pattern is transformed into polar coordinates relative to $\left(\mathrm{x}_{0}, \mathrm{y}_{0}\right)$ and divided into n sectors, as defined by the user. Here, it should be noted that the transformation involves conversion from discrete Cartesian $\left(\mathrm{x}_{\mathrm{i}}, \mathrm{y}_{\mathrm{i}}\right)$ coordinates to discrete polar coordinates $\left(q_{i}, \varphi_{i}\right)$. If this conversion is not performed correctly, artificial oscillations of the intensity can arise as a function of the azimuthal angle $\varphi_{i}$. To avoid this, for each pixel in Cartesian coordinates the radius from the centre is calculated and the intensity distributed to the polar pixels is weighted by the inter-pixel distance (cf. Fig. 2). This means that if a pixel in the Cartesian coordinates is at radial distance $\mathrm{q}_{i}+\Delta \mathrm{q}, \mathrm{q}_{\mathrm{i}}$ being an integer, the corresponding pixel in polar coordinates at $\mathrm{q}_{i}$ obtains (1- $\Delta q)$ times the original pixel intensity, whereas the pixel $\mathrm{q}_{i+1}$ gets $\Delta \mathrm{q}$ times the intensity. The same has to be considered for the distribution along $\varphi$ but is not checked since intensities are also integrated over the sectors during projection. The azimuthally integrated profiles are from here on represented by their central opening angle $\varphi_{\mathrm{j}}$. All intensities are finally normalized by the number of contributing pixels for each slot (again in weighted form).

The resulting line profiles $I\left(\varphi_{j}, q_{i}\right)$ are used to determine the peak position $q_{p e a k, j}$ of the radial intensity distribution in each sector with sub-pixel precision (cf. Fig. 3b). At the beginning of this step, the code smooths the profiles by a box blur algorithm using $1 \%$ of the radial boundaries given by the user selected ring mask as kernel size. It looks for the intensity minima $I\left(q_{\text {start }}\right)$ and $I\left(q_{\text {end }}\right)$, before and after the intensity maxima, respectively, inside these boundaries (cf. Fig. 3a). These minima are then used as new boundaries, $q_{s t a r t}$ and $q_{\text {end. }}$. A linear background is assumed from minima to minima and subtracted from the unsmoothed raw data. These background subtracted profiles $I_{b s u b}$ are then finally used to determine the maxima positions by a nonlinear least squares fit [19] using a pseudo-Voigt fit function [20] as model for the intensity distribution of the diffraction ring. This allows for accurate determination of peak positions as well as profile properties such as breadth and intensity. The fit minimizes the sum of squared distances for the radial pixels inside the boundaries by the following form:

$\sum_{i=s t a r t}^{\text {end }}\left(I_{b s u b}\left(q_{i}, \varphi_{j}\right)-p V\left(q_{i}\right)\right)^{2}=\min$

where

$p V(q)=I_{\text {peak }} \cdot\left(\frac{\eta}{\pi \omega}\left(1+\left(\frac{q-q_{\text {peak }}}{\omega}\right)^{2}\right)^{-1}+(1-\eta) \sqrt{\frac{\ln (2)}{\pi \omega^{2}}} \exp \left(-\ln (2)\left(\frac{q-q_{\text {peak }}}{\omega}\right)^{2}\right)\right)$

Here $\eta \in[0,1]$ is the mixing parameter, $2 \omega$ is the full width at half maximum (FWHM) of the distribution, $q_{\text {peak }}$ is the peak position and $I_{\text {peak }}$ is the peak intensity. By optimizing these parameters the minima for equation (2) is obtained. For each of the profile fits, the fit information, resulting parameters and the residual norm are saved into a spreadsheet for further analysis. 
Convergence of the nonlinear least squares fit is not guaranteed, and therefore only results consistent with the boundaries are considered further.

The maxima positions of all sectors $\left(q_{\text {peak }, j}, \varphi_{j}\right)$ are transformed back into contiguous Cartesian coordinates and an ellipse is fitted to the data points, again using a nonlinear least squares fit [21]. For the fit, an ellipse with the following parameter representation is used as a model function:

$\left(\begin{array}{l}x\left(\varphi_{k}\right) \\ y\left(\varphi_{k}\right)\end{array}\right)=\left(\begin{array}{l}x_{0} \\ y_{0}\end{array}\right)+Q(\alpha) \cdot\left(\begin{array}{l}a \cos \left(\varphi_{k}\right) \\ b \sin \left(\varphi_{k}\right)\end{array}\right)$

Here, $Q(\alpha)$ denotes the 2-dimensional rotation matrix and $\varphi_{\mathrm{k}} \in[0,2 \pi]$ the free parameter. The distance between the maxima positions and the parametric ellipse is then minimized as described in [21] giving a parameter vector $\mathbf{p}=\left(\mathrm{x}_{0}, \mathrm{y}_{0}, \mathrm{a}, \mathrm{b}, \alpha\right)$ that fully describes the ellipse. $\left(\mathrm{x}_{0}, \mathrm{y}_{0}\right)$ are the centre coordinates, $a$ and $b$ are the major and minor semi-axis and $\alpha$ is the angle between $a$ and the Cartesian x-axis. This process results in a precise location for the centre of the SAD pattern. The procedure is repeated iteratively for a specific number of times, taking the calculated centre from the previous iteration as the starting value for the next, to obtain the final coordinates of the centre. In the last step, the maxima positions $q_{\text {peak }}(\chi)=q_{1}(\chi)$ of each sector represented by its mean angle $\chi_{i}$ are calculated relative to the final centre in polar coordinates and saved. Figure $3 c$ shows an example of data points representing the $q_{1}(\chi)$ values and the corresponding elliptic fit. By taking these data points for a strained and a reference SAD pattern, the strain $\epsilon(\sigma, \chi)$ is calculated according to equation (1).

The 2-dimensional elastic strain tensor with respect to the coordinate axes of the SAD pattern is then calculated by fitting the $\epsilon$-values to the following equation [16]

$\epsilon(\sigma, \chi)=\epsilon_{11} \cdot \cos ^{2} \chi+\epsilon_{12} \cdot \sin \chi \cdot \cos \chi+\epsilon_{22} \cdot \sin ^{2} \chi$

Finally, the principal strains parallel $\left(e_{11}\right)$ and perpendicular $\left(e_{22}\right)$ to the straining axis are calculated from the components $\epsilon_{11}, \epsilon_{12}, \epsilon_{22}$ of the symmetrical elastic strain tensor by determining the eigenvalues. Additionally, the errors in the fit parameters are estimated by error propagation as well as by calculating the residual norm for the least square fits. It should be emphasized that although the atomic-level strain analysis is done in reciprocal space, the area of interest is selected in a TEM in real space with high accuracy by the SA aperture and the spatial resolution is given by its size. Therefore, local atomic-level strains of special amorphous regions, e.g. near crystalline inclusions can be quantitatively analyzed. 


\section{Experimental procedure}

\subsection{Sample preparation}

TiAl films (45 atomic \% Ti, 55 atomic $\% \mathrm{Al}$ ) with a thickness of $150 \mathrm{~nm}$ were synthesized by codeposition of $\mathrm{Ti}$ and $\mathrm{Al}$ on a 4" diameter, $200 \mu \mathrm{m}$ thick, (100) Silicon wafer by DC Magnetron Sputtering at a base pressure of $5 \times 10^{-8}$ Torr. The composition of the film was controlled by varying the input power of the individual sputtering guns containing 99.999\% pure Ti and Al targets. The film was amorphous in the as-deposited state. Photolithography and reactive Ion etching techniques were then used to co-fabricate MEMS based tensile testing stages having a built-in force and strain gauges along with dog-bone shaped freestanding film samples (Fig. 4). A detailed description of the process used in the fabrication of these devices can be found in $[22,23]$. The freestanding film samples had an effective gauge length of $395 \mu \mathrm{m}$ and a width of $30 \mu \mathrm{m}$.

\subsection{In-situ electron scattering experiments}

The MEMS devices containing the freestanding TiAl film samples were loaded in a straining TEM holder adapted for the special design of the device. The tensile tests were carried out in a Philips CM200 TEM using an accelerating voltage of $200 \mathrm{kV}$. The samples were uniaxially strained in steps of about $150 \mathrm{~nm}$. Bright-field images and selected area electron diffraction (SAD) patterns were recorded from the edge and the centre of the film at every strain step using a Gatan ${ }^{\text {TM }}$ Orius SC600 CCD camera with a 7 megapixel sensor. SAD patterns were taken from a circular area of diameter 1.2 micrometer using an exposure time of $10 \mathrm{sec}$. Before each SAD pattern was acquired, a normalization procedure was performed to reduce the magnetic remanence of the lenses so that the variation of the camera length in different SAD patterns was minimized. The illumination condition of the TEM was also kept constant during the deformation test. In order to obtain a high resolution in reciprocal space, which is beneficial for the strain analysis, a large camera length was chosen. In addition to SAD patterns taken from the same area, diffraction patterns were acquired from multiple locations along the width of the film to obtain information on the strain distribution at a given stress. The macroscopic stress and strain were measured by tracking the deflection of the built-in gauges (cf. Fig. 4) of the MEMS stage using an image correlation function in MATLAB ${ }^{\text {тм }}$. The distance between the gauges 1 and 2 gives the deformation on the sample while the relative deflection of gauge 2 with respect to the stationary gauge 3 gives the force acting on the sample. 


\section{Results and discussion}

4.1 Determination of strain tensor components from electron diffraction patterns of amorphous TiAl during in-situ tensile deformation

From a series of SAD patterns recorded from the same area at different stress levels during in-situ deformation of an amorphous TiAl film, a set of ellipse fits (as the one shown in Fig. 3c) were obtained. By using a reference image at zero stress the angular dependence of the strain $\varepsilon$ at a given stress is calculated according to equation (1). Figure 5 shows the measured strain values and the fitted $\epsilon(\sigma, \chi)$ curve for three different stress levels using equation (5). The corresponding macroscopic stresses $\sigma$ (parallel to the loading direction) were calculated from the force gauges of the MEMS device. The angle $\chi$ is defined with respect to the coordinate axis of the SAD pattern and the angular resolution of the sectors was chosen to be $1^{\circ}$. The $\varepsilon$ values vary from compressive strain at $\chi=62^{\circ}$ (minima) to tensile strain at $\chi=152^{\circ}$ (maxima). The maximum and minimum values of the curve corresponding to the principal strains, $e_{11}$ and $e_{22}$, increase with increasing macroscopic stress. It should be noted that the standard deviation of the fit decreases with decreasing angular resolution (due to averaging over a few degrees) and the corresponding principal strain values vary below $1 \times 10^{-4}$ only. Since the sample is subjected to uniaxial tensile stress, all the curves intersect at the same angle $\chi_{0}=87^{\circ}$ which corresponds to the zero strain direction. This angle $\chi_{0}$ is a function of the Poisson's ratio $v=-e_{22} / e_{11}$ of the material.

The principal strain values along the loading direction $\left(e_{11}\right)$ and the transverse direction $\left(e_{22}\right)$ are plotted as a function of stress in Figure 6. The strain value at a given stress is the mean of two evaluations based on two different reference images. Both $e_{11}$ and $e_{22}$ show a linear dependence on stress (as expected from Hooke's law) and reach $1 \%$ and $-0.17 \%$, respectively at the maximum stress. Using a linear fit for the variation of $e_{22}$ and $e_{11}$ over the entire loading, we obtained a Poisson's ratio of $v=0.23 \pm 0.02$. This value agrees well with macroscopic measurements using image correlations [24] and is reasonable for amorphous brittle metallic glasses [12]. It also shows a good agreement with results of polycrystalline $\gamma$-TiAl measured by resonant ultrasonic spectroscopy technique [25]. The Young's modulus (E) of the TiAl thin film calculated from the inverse slope of the $e_{11}-\sigma$ curve was found to be $185 \pm 2 \mathrm{GPa}$. The shear modulus G=75 $\pm 2 \mathrm{GPa}$ is calculated from $G=E / 2(1+v)$. The macroscopic strain values calculated from the gauges of the MEMS device are also shown in Figure 6 for comparison. These strain values show the same trend but are systematically higher compared to the atomic-level strain values obtained from SAD patterns and thus lead to a significantly lower Young's modulus ( $E=152 \pm 1 \mathrm{GPa}$ ). The difference in strains computed using two methods is considerably higher than the error in the atomic-level strain measurement (see discussion below) and can be attributed to the anelastic deformation 
resulting from topological rearrangements in metallic glasses $[10,14,24,26]$. Nevertheless, the Young's modulus (E=185 GPa) obtained by the diffraction method is within the modulus range (175-188 GPa) of polycrystalline TiAl [25], indicating that the diffraction method traces the atomic-level strain of the amorphous material whereas the macroscopic strain includes topological rearrangement of atoms as suggested previously $[8,14,27]$. Both Young's modulus values are lower compared to annealed, sputter deposited TiAl films calculated from temperature dependent internal stress measurements [28].

\subsection{Measurement of spatial strain variation}

In addition to SAD patterns taken from the same area at different stresses, diffraction patterns were recorded at a given stress from multiple locations of the film. Figure 7 shows the strain distribution along the width of the freestanding TiAl sample for a given external stress. The scan along the sample width shows variations of the atomic-level principal strain $e_{11}$ and a local minimum near the centre of the film. The strain variations are larger compared to the error of the method (cf. next paragraph) and indicate that the strain is not completely homogeneous across the sample width. Thus, by correlating the strain with the corresponding area, strain profiling or strain mapping can be carried out on a micrometer scale using this method. Note that the spatial resolution of the strain profiles/maps depends only on the size of the selected area apertures used to acquire the SAD patterns and hence can be varied as needed. It is also worth noting that the diffraction patterns are recorded at a given stress by moving the sample stage automatically to defined positions across the sample without changing the imaging mode. Hence, variations of the camera length due to remanence of the magnetic lenses are avoided.

\subsection{Reliability of strain measurement from electron diffraction patterns}

We have shown here that atomic-level elastic strain in amorphous materials can be measured directly from the position $q_{1}$ of the first intensity peak in electron diffraction images. It should be noted that the strain is derived from the change in the measured peak position of $I(q)$ and not of the structure function $S(q)$, where the average atomic scattering factor has to be taken into account. However, the results are expected to be the same for both cases (as shown in [8]) since the peak shift due to elastic straining is small and the $q$-dependence of the atomic scattering factor is negligible. This strain evaluation method is advantageous with respect to complementary real space methods (based on calculation of pair distribution functions), because it requires the accurate measurement of the peak position only. For the pair distribution functions the evaluation has to be done over the full reciprocal space, including fits to the background at high q values and low intensities. 
The elastic strain determination is based on the accurate analysis of a strained SAD pattern with respect to a reference (circular) one. But unlike an X-ray diffractometer, the scattering geometry is not fixed in a TEM and hence an error is introduced when the camera length varies between different SAD images. In order to minimize this error to $<1 \%$ a normalization procedure of the magnetic lens system was performed before each SAD exposure. Still, a small variation of the camera length between consecutive SAD images can lead to a change of the $e_{11} / e_{22}$ ratio and to a vertical shift of the $\epsilon(\sigma, \chi)$ curve. Therefore, variations in the camera length can be traced by abrupt changes in the Poisson's ratio $v$ and $\chi_{0}$ (angle corresponding to the strain free direction). In addition to external stresses leading to anisotropic SAD pattern, internal stresses in thin films as well as astigmatism of lenses can lead to slight distortions in the diffraction pattern. Although astigmatism can be corrected in a TEM, some residual astigmatism may be present in the diffraction pattern. Both deviations from circular symmetry (astigmatism and internal stresses) are included in the reference pattern used to calculate the strain and influence the absolute strain values but not the relative ones at different stress states.

We also quantified the precision and accuracy of this method of strain measurement. Strain precision (or strain sensitivity) corresponds to the strain level within an unstrained area and can be viewed as the noise inherent in the technique [1]. From the evaluation of 10 SAD images, a strain precision of $2 \times 10^{-4}$ was estimated. Since amorphous materials can have different levels of internal stresses, strain precision can vary between different thin film samples. In order to estimate the strain accuracy, the deviation of the measured strains from the actual strain values was calculated using simulated SAD images with known distortions. In our simulations the intensity profile of the first diffuse SAD ring was taken to be a Gaussian curve as a first approximation. The geometric parameters (ring diameter and ring width with respect to pixel size), intensity and noise level of the simulated pattern was set close to those of the experimental diffraction patterns. Table 1 shows parameters of two simulated distorted rings representing different strain values and the parameters determined from the ellipse fit using equations (1) (3). In addition to the position of the centre $\left(x_{0}, y_{0}\right)$ and the parameters of the ellipse $(a, b, \alpha)$, the corresponding principal strain values $\mathrm{e}_{11}$ and $\mathrm{e}_{22}$ are shown. For the two given images, the fitted values were very close to the actual ones indicating a strain accuracy of about $1 \times 10^{-4}$. From the measurement of four sets of distorted SAD patterns, the strain accuracy was calculated in the form of the relative error as shown in Fig. 8. In the figure, full and open symbols refer to simulations of images showing a standard deviation of the noise of 5 and $10 \%$ of the intensity maximum, respectively. The relative error decreases with increasing magnitude of the principal strain values, $e_{11}$ and $e_{22}$, and is below $3 \%$; the absolute strain accuracy is still $<1 \times 10^{-4}$. 


\section{Conclusion}

Atomic-level elastic strain in amorphous materials can be measured accurately from the position of the intensity peak of the first diffuse ring in selected area electron diffraction images acquired in a TEM. In contrast to the macroscopically measured elastic strain, atomic-level elastic strain obtained from reciprocal space measurements is not influenced by deformation induced atomic rearrangements of amorphous materials. Thus, macroscopic elastic strain and atomic-level elastic strain carry different information. Although the analysis is conducted in reciprocal space the area of interest $\left(<1 \mu \mathrm{m}^{2}\right)$ from which the atomic-level strain is assessed can be selected in real space with high accuracy. Therefore, the local strain of special amorphous regions, e.g. near crystalline inclusions can be quantitatively analyzed.

Macroscopic stress and atomic-level strain measured during in-situ tensile deformation of an amorphous TiAl film showed a linear correlation as expected from Hooke's law. Nevertheless, the atomic-level strain was lower compared to the macroscopic strain, most likely due to anelasticity. In addition, the full 2-dimensional strain tensor was assessed from the diffraction pattern analysis and the elastic properties of the sample was calculated by taking external stresses into account. The capability of the method to construct strain profiles/maps with micrometer scale resolution was also demonstrated by plotting the strain variation along the sample width. From the evaluation of simulated diffuse diffraction rings with known distortions a strain accuracy of about $1 \times 10^{-4}$ is estimated for the present analysis.

The determination of atomic-level elastic strain necessitates an accurate analysis of the electron diffraction pattern which is implemented via plug-ins to the GATAN ${ }^{\mathrm{TM}}$ Digital-Micrograph platform. The code (i) accurately determines the centre by an elliptic fit of the distorted first ring, (ii) calculates the position of the intensity maxima with sub-pixel precision and (iii) computes the atomic-level strain by comparing the elliptically distorted pattern (induced by strain) with a reference pattern. The developed software code [15] can be used freely provided this publication is appropriately referenced.

\section{Acknowledgement}

The authors would like to gratefully acknowledge the use of facilities at the John M. Cowley Centre for High Resolution Electron Microscopy and the Centre for Solid State Electronics Research at Arizona State University and at the Faculty of Physics of the University of Vienna. We thank S. Noisternig for the provision of a Mathematica code to calculate intensity rings of known distortions. C. E. and C. R. acknowledge financial support by the Austrian Science Fund 
FWF:[11309]. R. S. and J. R. acknowledge funding from the National Science Foundation (NSF) grants CMMI 1400505 and DMR 1454109.

\section{References}

[1] A. Béché, J.L. Rouvière, J.P. Barnes, D. Cooper, Strain measurement at the nanoscale: Comparison between convergent beam electron diffraction, nano-beam electron diffraction, high resolution imaging and dark field electron holography., Ultramicroscopy. 131 (2013) 10-23. doi:10.1016/j.ultramic.2013.03.014.

[2] C. Mahr, K. Müller-Caspary, T. Grieb, M. Schowalter, T. Mehrtens, F.F. Krause, et al., Theoretical study of precision and accuracy of strain analysis by nano-beam electron diffraction., Ultramicroscopy. 158 (2015) 38-48. doi:10.1016/j.ultramic.2015.06.011.

[3] F. Hüe, M. Hÿtch, H. Bender, F. Houdellier, A. Claverie, Direct Mapping of Strain in a Strained Silicon Transistor by High-Resolution Electron Microscopy, Phys. Rev. Lett. 100 (2008) 156602. doi:10.1103/PhysRevLett.100.156602.

[4] A. Béché, J.L. Rouvière, J.P. Barnes, D. Cooper, Dark field electron holography for strain measurement, Ultramicroscopy. 111 (2011) 227-238.

doi:10.1016/j.ultramic.2010.11.030.

[5] K. Usuda, T. Numata, T. Irisawa, N. Hirashita, S. Takagi, Strain characterization in SOI and strained-Si on SGOI MOSFET channel using nano-beam electron diffraction (NBD), Mater. Sci. Eng. B Solid-State Mater. Adv. Technol. 124-125 (2005) 143-147. doi:10.1016/j.mseb.2005.08.062.

[6] L. Clément, R. Pantel, L.F.T. Kwakman, J.L. Rouvière, Strain measurements by convergentbeam electron diffraction: The importance of stress relaxation in lamella preparations, Appl. Phys. Lett. 85 (2004) 651-653. doi:10.1063/1.1774275.

[7] H.F. Poulsen, J. a. Wert, J. Neuefeind, V. Honkimäki, M. Daymond, Measuring strain distributions in amorphous materials, Nat. Mater. 4 (2004) 33-36. doi:10.1038/nmat1266.

[8] T.C. Hufnagel, R.T. Ott, J. Almer, Structural aspects of elastic deformation of a metallic glass, Phys. Rev. B. 73 (2006) 064204. doi:10.1103/PhysRevB.73.064204.

[9] M. Stoica, J. Das, J. Bednarcik, H. Franz, N. Mattern, W.H. Wang, et al., Strain distribution in $\mathrm{Zr}_{64.13} \mathrm{Cu}_{15.75} \mathrm{Ni}_{10.12} \mathrm{Al}_{10}$ bulk metallic glass investigated by in situ tensile tests under synchrotron radiation, J. Appl. Phys. 104 (2008) 013522. doi:10.1063/1.2952034.

[10] W. Dmowski, T. Iwashita, C.-P. Chuang, J. Almer, T. Egami, Elastic Heterogeneity in Metallic Glasses, Phys. Rev. Lett. 105 (2010) 2-5. doi:10.1103/PhysRevLett.105.205502.

[11] M. Ashby, A.L. Greer, Metallic glasses as structural materials, Scr. Mater. 54 (2006) 321326. doi:10.1016/j.scriptamat.2005.09.051. 
[12] A.L. Greer, Metallic glasses...on the threshold, Mater. Today. 12 (2009) 14-22. doi:10.1016/S1369-7021(09)70037-9.

[13] C. Schuh, T. Hufnagel, U. Ramamurty, Mechanical behavior of amorphous alloys, Acta Mater. 55 (2007) 4067-4109. doi:10.1016/j.actamat.2007.01.052.

[14] T. Egami, T. Iwashita, W. Dmowski, Mechanical Properties of Metallic Glasses, Metals (Basel). 3 (2013) 77-113. doi:10.3390/met3010077.

[15] C. Ebner, SAD-strain, (2015). http://www.univie.ac.at/elmi-software/.

[16] N. Mattern, J. Bednarčik, S. Pauly, G. Wang, J. Das, J. Eckert, Structural evolution of Cu-Zr metallic glasses under tension, Acta Mater. 57 (2009) 4133-4139. doi:10.1016/j.actamat.2009.05.011.

[17] C. Sanderson, P.O. Box, S. Lucia, Armadillo : An Open Source C ++ Linear Algebra Library for Fast Prototyping and Computationally Intensive Experiments Technical Report, (2011).

[18] C. Gammer, C. Mangler, C. Rentenberger, H.P. Karnthaler, Quantitative local profile analysis of nanomaterials by electron diffraction, Scr. Mater. 63 (2010) 312-315. doi:10.1016/j.scriptamat.2010.04.019.

[19] J. Wuttke, lmfit - a C library for Levenberg-Marquardt least-squares minimization and curve fitting. Version 5.1, retrieved on 14th April 2015 from http://apps.jcns.fzjuelich.de/lmfit.

[20] P. Thompson, D.E. Cox, J.B. Hastings, Rietveld Refinement of Debye-Scherrer Synchrotron X-ray Data from A1203, J. Appl. Crystallogr. 20 (1987) 79-83. doi:10.1107/S0021889887087090.

[21] W. Gander, G.H. Golub, R. Strebel, Least-squares fitting of circles and ellipses, Bit. 34 (1994) 558-578. doi:10.1007/BF01934268.

[22] J.H. Han, M.T.A. Saif, In situ microtensile stage for electromechanical characterization of nanoscale freestanding films, Rev. Sci. Instrum. 77 (2006) 045102. doi:10.1063/1.2188368.

[23] W. Kang, J. Rajagopalan, M.T. A. Saif, In Situ Uniaxial Mechanical Testing of Small Scale Materials-A Review, Nanosci. Nanotechnol. Lett. 2 (2010) 282-287. doi:10.1166/nnl.2010.1107.

[24] R. Sarkar, C. Ebner, E. Izadi, C. Rentenberger, J. Rajagopalan, submitted, (2016).

[25] Y. He, R.B. Schwarz, A. Migliori, Elastic constants of single crystal g - TiAl, J. Mater. Res. 10 (1995) 1187-1195.

[26] T.C. Hufnagel, R.T. Ott, J. Almer, Structural aspects of elastic deformation of a metallic glass, Phys. Rev. B. 73 (2006) 064204. doi:10.1103/PhysRevB.73.064204.

[27] Y. Suzuki, T. Egami, Shear deformation of glassy metals: Breakdown of Cauchy relationship and anelasticity, J. Non. Cryst. Solids. 75 (1985) 361-366. 
[28] M. Chinmulgund, R.B. Inturi, J. A. Barnard, Effect of Ar gas pressure on growth, structure, and mechanical properties of sputtered Ti, Al, TiAl, and Ti3Al films, Thin Solid Films. 270 (1995) 260-263. doi:10.1016/0040-6090(95)06990-9.

\begin{tabular}{|l|l|c|c|c|c|c|c|c|}
\hline & $\#$ & \multicolumn{2}{|c|}{ position of the centre } & \multicolumn{3}{|c|}{ ellipse } & \multicolumn{2}{c|}{ principal strain } \\
\hline & & $\mathrm{x}_{0}$ [pixel] & $\mathrm{y}_{0}$ [pixel] & $\mathrm{a}$ [pixel] & $\mathrm{b}$ [pixel] & angle $\alpha$ [ $\left.{ }^{\circ}\right]$ & $e_{11}$ & $e_{22}$ \\
\hline \multirow{2}{*}{$\begin{array}{l}\text { actual } \\
\text { values }\end{array}$} & 1 & 1254.5 & 1392.2 & 619.8 & 611.8 & 124 & 0.010134 & -0.0029 \\
\cline { 2 - 9 } & 2 & 1254.5 & 1392.2 & 621.5 & 605.8 & 124 & 0.020139 & -0.00563 \\
\hline $\begin{array}{l}\text { values } \\
\text { from fit }\end{array}$ & 1 & 1254.501 & 1392.208 & 619.77 & 611.77 & 123.9 & 0.010166 & -0.00287 \\
\cline { 2 - 9 } & 2 & 1254.529 & 1392.184 & 621.47 & 605.76 & 124.0 & 0.020219 & -0.00557 \\
\hline
\end{tabular}

Table 1: Comparison of parameters from two simulated distorted SAD patterns representing different atomic-level elastic strains with those obtained from the data fitting procedure. 


\section{Figures}
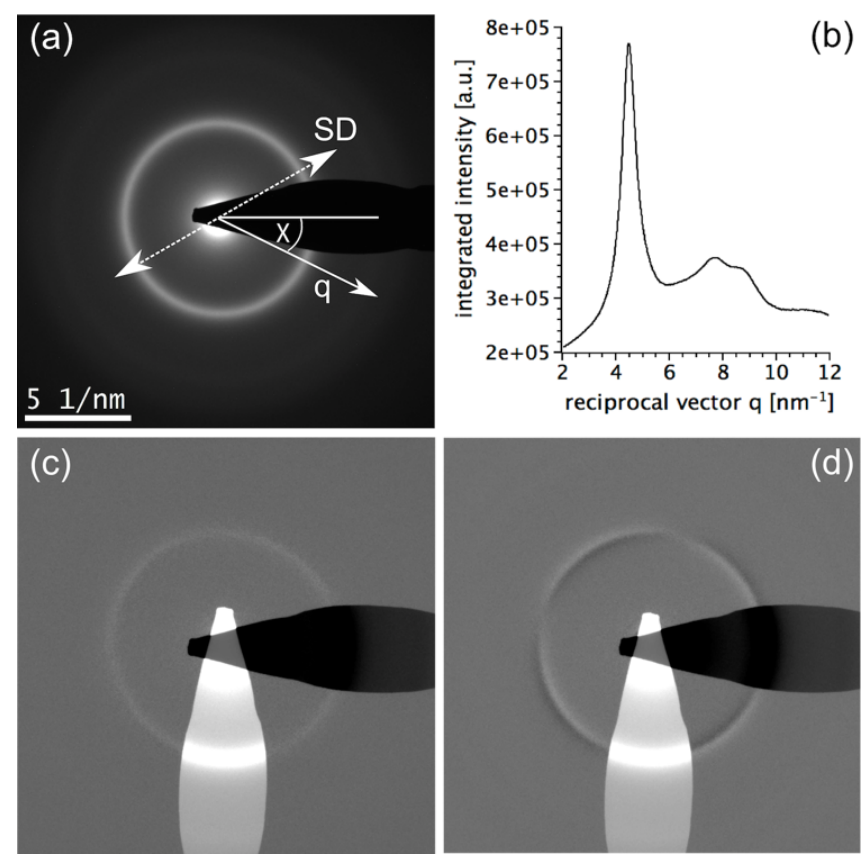

Figure 1: Selected area electron diffraction (SAD) pattern and the average image of SAD patterns with their rotated and inverted counterparts. (a) SAD pattern of a TiAl thin film showing the first intense amorphous ring and the corresponding reciprocal lattice vector $\boldsymbol{q}$ at an angle $\chi$. The straining direction (SD) is indicated in the image by the double arrow. (b) Intensity profile $I(q)$ obtained by integration of the intensity along rings. (c) SAD pattern overlay at zero stress reveals a circular symmetric diffuse amorphous ring. (d) Due to the ellipticity of the SAD pattern of the strained sample, a clear deviation from circular symmetry can be observed in the image overlay.

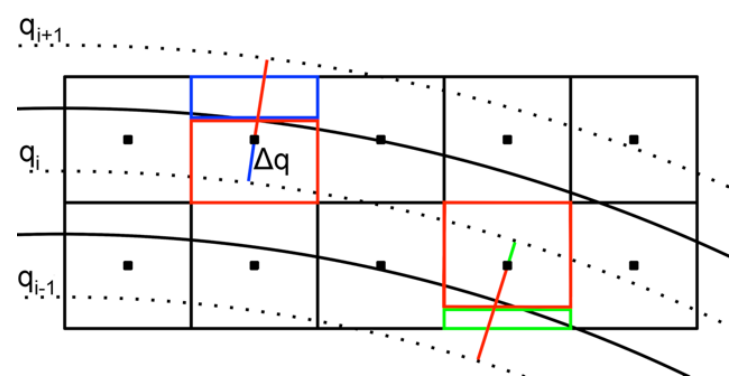

Figure 2. Polar transformation of the discrete intensities: Schematic representation of the polar transformation used in the code. The distances of each pixel centre is measured relative to the assumed centre of the ellipse. The area of a cartesian pixel at distance $\mathrm{q}_{\mathrm{i}}+\Delta \mathrm{q}$ can partially overlap with a polar pixel $\mathrm{q}_{\mathrm{i}+1}$. Therefore, the intensity weighted by the sub-pixel distance $\Delta \mathrm{q}$ (as indicated by the coloured boxes) is added to the polar pixel $\mathrm{q}_{\mathrm{i}+1}$, whereas the rest (intensity weighted by (1$\Delta q)$ ) is added to the polar pixel $q_{i}$. Cases where the cartesian pixel overlaps with 3 polar pixels are not considered since the error is small. This weighting is not performed in the angular direction since intensities are integrated over several red boxes composing one sector. 

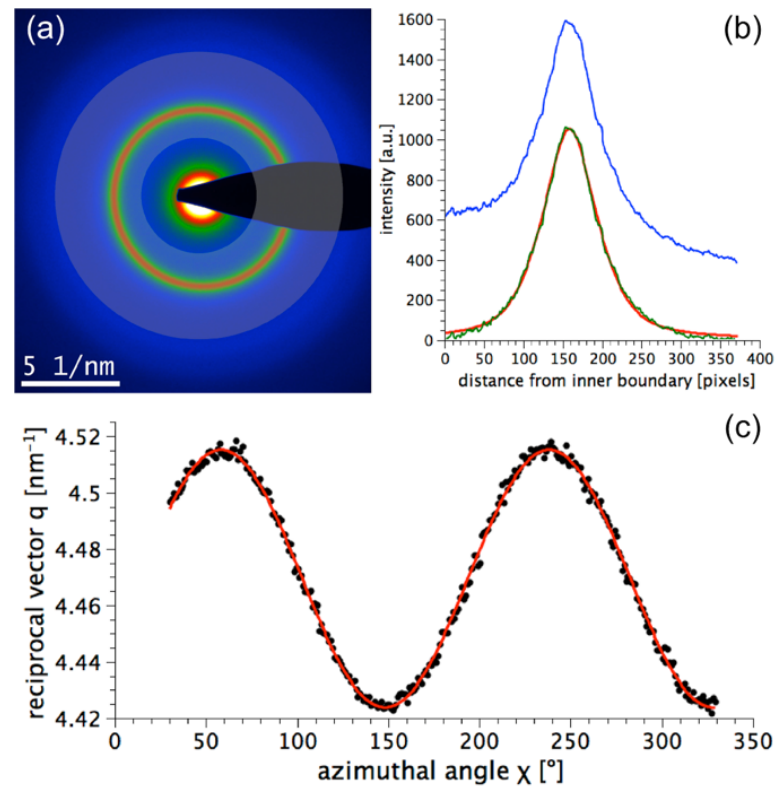

Figure 3. SAD pattern, intensity profiles and ellipse fit: (a) Using the Digital Micrograph ${ }^{\mathrm{TM}} \mathrm{script}$ described in this work, intensity profiles as a function of the azimuthal angle $\chi$ are extracted between a minimum and maximum $q$ value defined by the mask. (b) The experimental intensity profile (raw data in blue) subtracted by the background is denoted by the green curve and the corresponding fitted function is shown in red. The range of the pixel values reflects the minimum and maximum $q$ values of the mask shown in (a). (c) A plot showing the $q_{1}$-positions of the maxima of the experimental intensity profiles at different angles $\chi$ along the SAD ring. The red curve represents the best fit of an ellipse. The $q$ values are larger perpendicular to the tensile loading direction and smaller parallel to it.

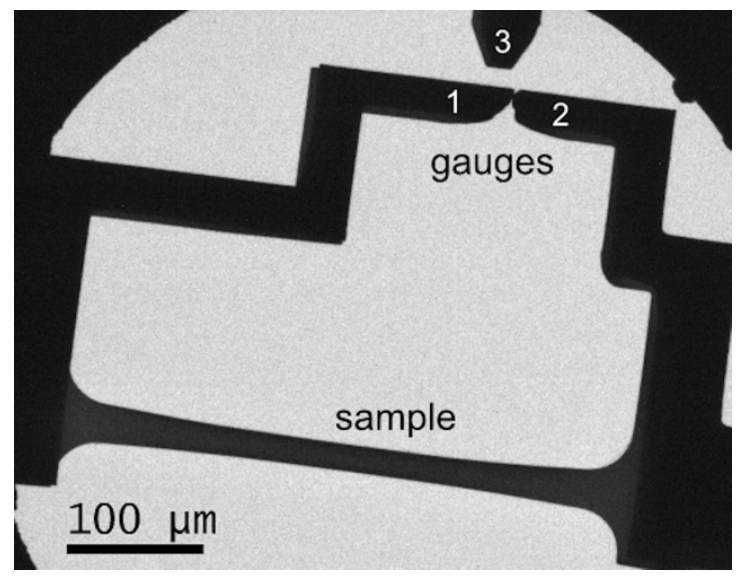

Figure 4. MEMS device and thin film sample: TEM image of the freestanding TiAl thin film and of the strain (1-2) and force-sensing gauges (2-3) facilitating the measurement of the macroscopic strain and stress. 


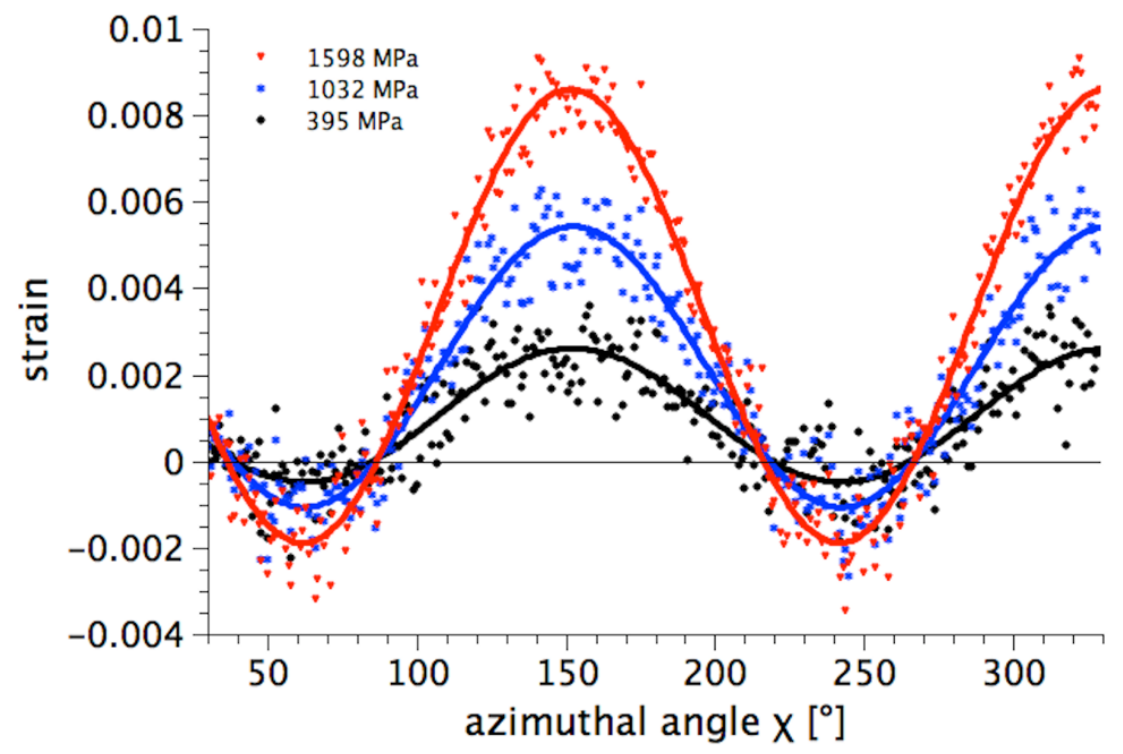

Figure 5: Angular dependence of atomic-level strain as a function of stress at various stages of deformation. The full lines denote fits of the experimental data using equation (3). The maximum and minimum of a curve corresponds to the principal strains $e_{11}$ and $e_{22}$, respectively.

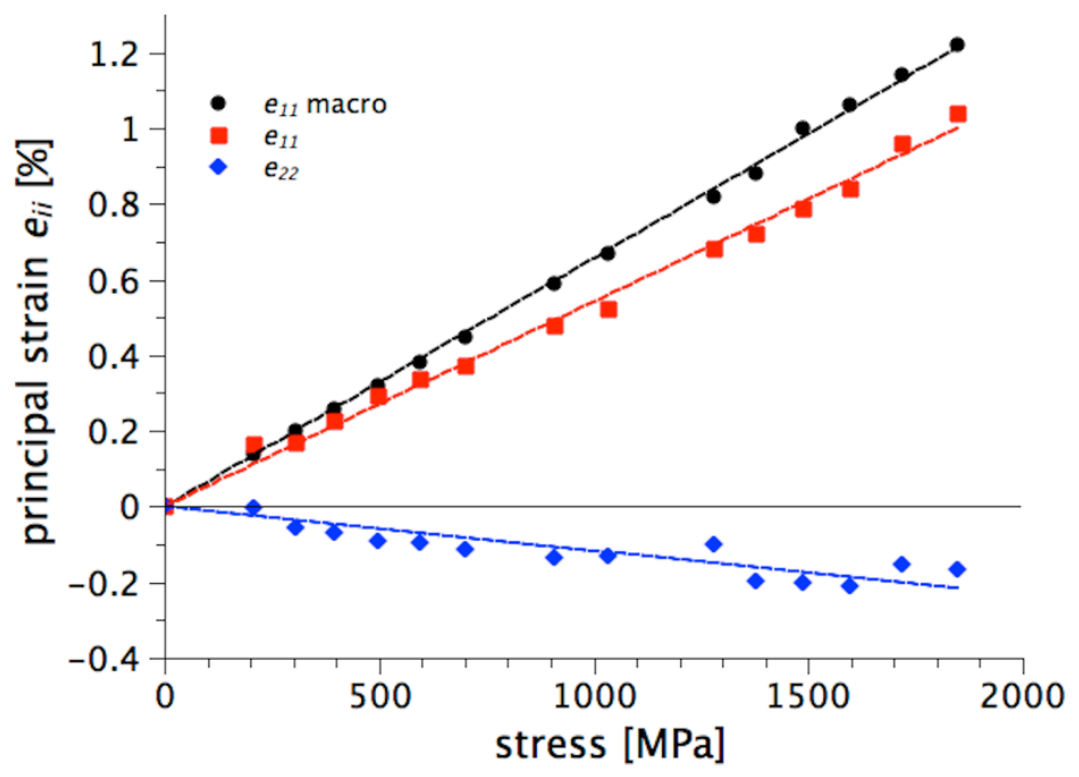

Figure 6. Curves of principal strains $e_{11}$ and $e_{22}$ as a function of stress obtained from in-situ measurements of amorphous TiAl: The calculated atomic-level principal strain along the loading direction $\left(e_{11}\right)$ shows a linear dependence on stress as expected for an elastic tensile deformation. Due to the Poisson effect, the calculated atomic-level principal strain along the transverse direction $\left(e_{22}\right)$ shows a negative slope. The dashed lines represent the line of best fit for the data points. The macroscopic strain curve measured from the strain gauges shows a line with a different slope compared to that of $e_{11}$. This discrepancy is caused by the anelastic deformation of the material [24]. 


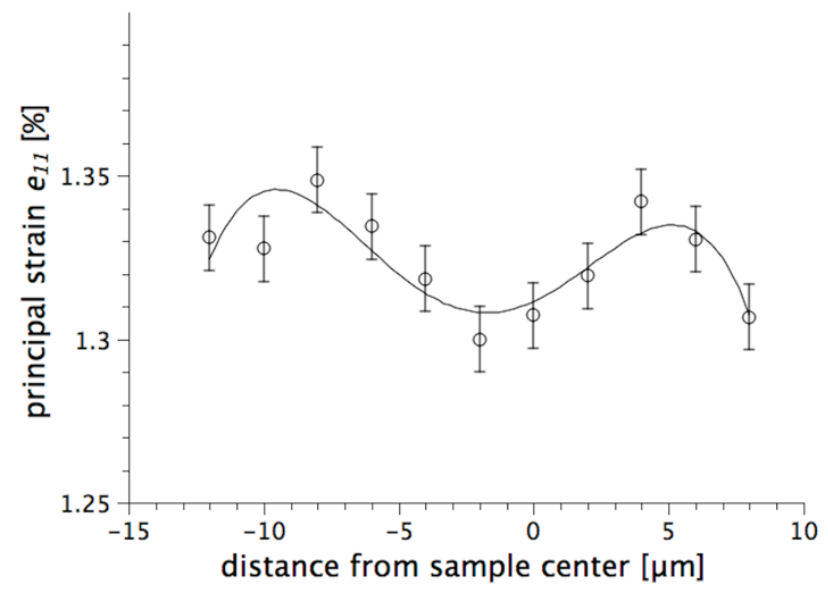

Figure 7. Strain profile: The atomic-level principal strain along the loading direction $\left(e_{11}\right)$ measured across the sample width. The line scan shows a variation in strain at a given external stress. The error bars reflect the accuracy of the method. A polynomial fit to the data is included to guide the eye.

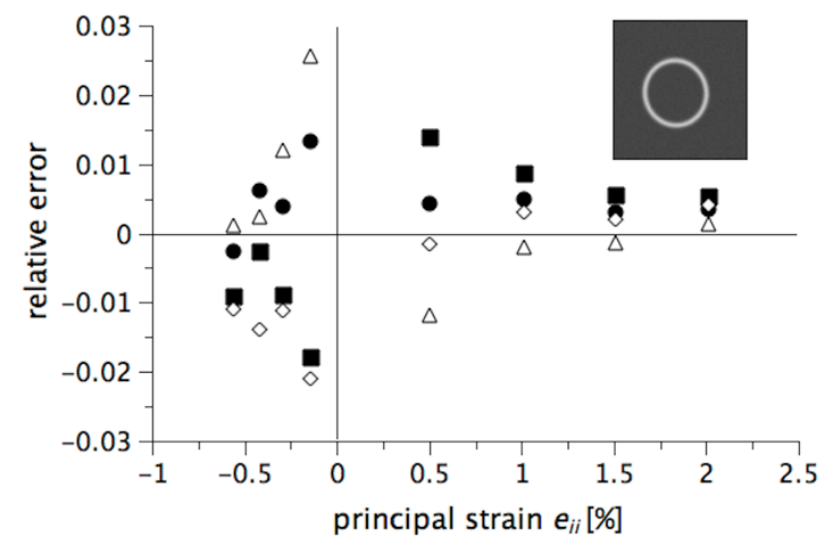

Figure 8. Error evaluation from simulated SAD patterns: Plot of the relative strain error deduced from strain measurements of simulated SAD patterns with known distortions using the Digital Micrograph $^{\mathrm{TM}}$ plug-ins described in this paper. Full and open symbols refer to patterns with different levels of noise. The inset shows an example of a simulated SAD pattern containing one diffuse diffraction ring. 